\title{
Lithiation of toluene by organolithium compounds activated by lithium polyether alkoxides
}

\author{
N. Nugay ${ }^{(1)}$, T. Nugay $^{(1)}$, R. Jérôme ${ }^{(2)}$, Ph. Teyssié ${ }^{(2)}$ \\ (1) Department of Chemistry, Polymer Research Centre, Bogazici University, 80815 Istanbul, Turkey \\ (2) Centre for Education and Research on Macromolecules (CERM), University of Liège, 4000 Liège, Belgium
}

\begin{abstract}
The catalytic effect of polyether alkoxides (PEAs) on the activity of organolithium compounds as metallating agent has been investigated. Toluene has been lithiated by using different organolithiums activated by various PEAs. It has been found that $n$-BuLi, yields higher conversion with $100 \%$ benzylic position metallation, whereas $s e c-\mathrm{BuLi}$ gives some metallation at ring positions with the most reactive ligand, lithium 2-methoxy ethoxide (LiOEM). Optimum PEA to organolithium molar ratio for both sec-and $n$-BuLi activated by LiOEM where the amount of toluene being kept constant, was found to be as 3:1 with highest yield and regioselectivity. Substantial increment in yield was observed when the amount of substrate increased whereas regioselectivity stayed almost constant. It was also found that, for a constant reactant molar ratio $(3: 1: 15)$ for which the optimum results were achieved, reaction began early and then yield values increased with time whereas upon increase in reaction temperature from -78 to $20{ }^{\circ} \mathrm{C}$, the benzylic metallation yield increased drastically while keeping the same regioselectivity high.
\end{abstract}

Keywords: Lithiation of toluene; Polyether alkoxide; Organolithium compounds; Regioselectivity

\section{Introduction}

Metallation of alkyl benzenes, i.e. toluene, using organosodium and potassium compounds, has received considerable attention in the chemical literature, resulting in a variety of mechanistic interpretations [1-4]. Such workers have made Very significant advances in the area of base catalysed exchange of hydrocarbons as Shatenstein [5], Streitwieser et al. [6] and an Esso research group [7].

Later, it has been found that mixtures of organolithiums with alkoxides of heavier alkali metals (diversely known as LICKOR reagent, Lochmann's base or Schlosser's base) are very reactive agents for metallation of hydrocarbons, even more reactive than conventional butyl lithium electron donor complexes [8-13], which make them efficient also in polymer chemistry as well as organic chemistry [12]. These super bases exhibit unprecedented reactivity and at the same time interesting selectivity profiles, especially when they are applied to the metallation of straight-chain and branched olefins [10,11]. Recently, still higher yields and higher reaction rates as well as a more selective metallation in the side chain of alkyl benzenes were achieved by Lochmann and Petranek [14], using super bases which contain an enhanced amount of an alkoxide with more bulky groups than tert-butoxide, and are named as second generation super bases.

On the other hand, metallation of these alkylaromatics with an organolithium was generally unsuccessful. Hein et al. [15] have prepared benzyl lithium from dibenzyl mercury and ethyl lithium, whereas Ziegler and Dersch

[16] have used two different approaches for the lithiation of toluene, i.e. simple interaction of benzyl magnesium chloride in ether with metallic lithium, or a two stage reaction between benzyl chloride and phenyl lithium. None of these procedures is quite satisfactory, due to the involving of difficulty accessible dibenzyl mercury, long reaction time, and erratic occurrence of a mixture of two different, but relatively reactive organometallic compounds.

Later on, it has been discovered that tertiary amines activate alkyl lithium compounds [17] in lithiation reactions of alkyl benzene, resulting in behaviour similar to that of alkyl sodium [18]. Using either diazobicyclo octane (DABCO) [5,5] or $N, N, N^{\prime}, N^{\prime}$-tetramethylenediamine, Eberhard and Butte [19] found that toluene undergoes quantitative metallation with $n$-BuLi to give benzyl lithium.

Recently, a patent [20] was issued dealing with the lithiation of toluene in the presence of polycyclic aromatics and conjugated dienes, resulting in benzyl-lithium with $97 \%$ yield.

In this work, it has been tried to determine whether introduction of a polyether lithium alkoxide, already used in nucleophilic polymerisation [13], increases the activity of an organolithium compound as metallating agent. A special attention has been paid to the effect of the molar ratio of reaction components, reaction time, basicity and 
bulkiness of polyether alkoxides (PEAs) used, and difference in the organolithium structure on the lithiation process as well as reaction temperature.

\section{Experimental}

\subsection{Materials}

$n$-BuLi was purchased from Janssen as ca. 2.5 M solution in hexane. Diphenyl methyl lithium (DPMLi) was prepared by the reaction of diphenyl methane and lithium naphthalene in THF, at room temperature for $24 \mathrm{~h}$. Lithium naphthalene was prepared by reaction of lithium metal with naphthalene in THF at the same temperature. Diphenyl hexyl lithium (DPHLi) resulted from the reaction of $n$-BuLi with diphenylethylene in toluene, also at room temperature for $24 \mathrm{~h}$. Toluene was first refluxed over $\mathrm{CaH}_{2}$ and finally distilled over oligo(styryl lithium) just prior to use. Lithium PEAs listed in Table 1 were prepared by reaction of an excess amount of lithium with the parent alcohol in cyclohexane.

After refluxing of alcohol in THF in the presence of excess lithium metal for $24 \mathrm{~h}$, THF was distilled off under reduced pressure and replaced by cyclohexane. The metal in excess was filtered through a series of $F$ type finest sintered glass under argon atmosphere. Methyl iodide and sec-BuLi were the product of Janssen and used as received.

\subsection{Metallation reactions}

All lithiation reactions were conducted in a flamed glass reactor under a nitrogen atmosphere. Reagents were transferred through syringe or stainless steel capillaries. Reactions were carried out at different temperatures. Gas chromatographic separations were performed on a Varian Star 3400 CX chromatograph using a fused silica capillary column of $30 \mathrm{~m}$ length packed with PDMS (5\% phenyl).

A solution of $0.0025 \mathrm{~mol}$ of organolithium was added dropwise to a solution of 0.0075 mol of PEA (i.e. LiOEM, $0.8 \mathrm{M}$ in cyclohexane) and a given amount of toluene (i.e. $0.0375 \mathrm{~mol}$ ) as a substrate through a rubber septum by means of a syringe.

Table 1 PEAs Used

\begin{tabular}{lll}
\hline Lithium 2-(2-methoxyethoxy) ethoxide & $\mathrm{LiOCH}_{2} \mathrm{CH}_{2} \mathrm{OCH}_{2} \mathrm{CH}_{2} \mathrm{OCH}_{3}$ & LiOEEM \\
Lithium 2-methoxy ethoxide & $\mathrm{LiOCH}_{2} \mathrm{CH}_{2} \mathrm{OCH}_{3}$ & LiOEM \\
Lithium(dimethylamino) ethoxide & $\mathrm{LiOCH}_{2} \mathrm{CH}_{2} \mathrm{~N}_{3}\left(\mathrm{CH}_{3}\right)_{2}$ & $\mathrm{LiOEN(Me})_{2}$ \\
Sodium 2-(2-methoxyethoxy) ethoxide & $\mathrm{NaOCH}_{2} \mathrm{CH}_{2} \mathrm{OCH}_{2} \mathrm{CH}_{2} \mathrm{OCH}_{3}$ & $\mathrm{NaOEEM}$ \\
Lithium 2-(2-butoxyethoxy) ethoxide & $\mathrm{LiOCH}_{2} \mathrm{CH}_{2} \mathrm{OCH}_{2} \mathrm{CH}_{2} \mathrm{OC}_{4} \mathrm{H}_{9}$ & LiOEEBu \\
\hline
\end{tabular}

Soon after adding the organolithium, the mixture becomes heterogeneous and remained so throughout the metallation time at constant temperature. Reaction was completed by stirring the mixture for the indicated period. After cooling to $-50^{\circ} \mathrm{C}$ Varian, a two molar excess of $\mathrm{CH}_{3} \mathrm{I}$ was rapidly added to the solution. The mixture was left overnight (shorter quenching time was also used but this had no influence on the results), then LiI was removed by filtration. The structure of soluble reaction products was determined by gas chromatography by comparison with available standards such as ethyl benzene, $m-, o-, p$-xylene, and mesitylene that were separately recorded. All chromatograms were obtained from $2 \%$ (vol.\%) solutions in chloroform. 


\section{Results and discussion}

A set of reactions was conducted to see the effect of PEA structure on the metallation process by $s e c-\mathrm{BuLi}$, or, $n$ BuLi systems (Table 2).

Table 2

Lithiation of toluene using different organolithium activated by various PEAs ${ }^{a}$

\begin{tabular}{|c|c|c|c|c|}
\hline \multirow[t]{2}{*}{ PEA } & \multirow[t]{2}{*}{ Organolithium (Rli) } & \multirow[t]{2}{*}{ Yield $(\mathrm{mol} \%)^{b}$} & \multicolumn{2}{|c|}{$\mathrm{GC}$ after reaction with $\mathrm{CH}_{3} \mathrm{I}$} \\
\hline & & & Benzylic $^{\mathrm{c}}$ & Ring $^{\mathrm{d}}$ \\
\hline $\mathrm{NaOEEM}^{\mathrm{e}}$ & $s e c-\mathrm{BuLi}$ & - & - & - \\
\hline LiOEEM $^{\mathrm{e}}$ & $s e c-\mathrm{BuLi}$ & - & - & - \\
\hline $\mathrm{LiOEEBu}^{\mathrm{e}}$ & $s e c-\mathrm{BuLi}$ & - & - & - \\
\hline $\operatorname{LiOEN}(\mathrm{Me})_{2}{ }^{\mathrm{e}}$ & $s e c-\mathrm{BuLi}$ & 13.0 & 81.0 & 19.0 \\
\hline $\mathrm{LiOEM}^{\mathrm{f}}$ & $s e c-\mathrm{BuLi}$ & 46.0 & 98.0 & 1.5 \\
\hline $\mathrm{NaOEEM}^{\mathrm{f}}$ & $n$-BuLi & 1.2 & 29.0 & 71.0 \\
\hline LiOEEM $^{\mathrm{f}}$ & $n-\mathrm{BuLi}$ & 5.0 & 100.0 & - \\
\hline $\mathrm{LiOEEBu}^{\mathrm{f}}$ & $n$-BuLi & 6.2 & - & 100.0 \\
\hline $\operatorname{LiOEN}(\mathrm{Me})_{2}{ }^{\mathrm{f}}$ & $n-\mathrm{BuLi}$ & 36.0 & 100.0 & - \\
\hline $\mathrm{LiOEM}^{\mathrm{f}}$ & $n$-BuLi & 73.0 & 100.0 & - \\
\hline $\mathrm{LiOEM}^{\mathrm{f}}$ & $\mathrm{DPMLi}^{\mathrm{g}}$ & - & - & - \\
\hline $\mathrm{LiOEM}^{\mathrm{f}}$ & DPMLi $^{\mathrm{h}}$ & - & - & - \\
\hline
\end{tabular}

${ }^{a}$ Reaction time, $24 \mathrm{~h}$; reaction temperature, $20^{\circ} \mathrm{C}$.

${ }^{b}$ Calculated with respect to organolithium.

${ }^{c}$ Mainly ethylbenzene.

${ }^{d} o-, m-, p$-Xylene and mesitylene.

e PEA:RLi:toluene, 1.3:1:6.5

${ }^{f}$ PEA:RLi:toluene, $3: 1: 15$.

${ }^{g}$ Diphenylmethyllithium.

${ }^{h}$ Diphenylhexyllithium.

It is clear from the table that, sec-BuLi cannot efficiently metallate toluene in the presence of NaOEEM, LiOEEM, and LiOEEBu type PEAs, since these alkoxides form sterically hindered chelated systems with sec$\mathrm{BuLi}$ which then inhibit the metallation reaction under these conditions. $\mathrm{LiOEN}(\mathrm{Me})_{2}$ is still effective in metallation because of the less coordinated character of the structure that can cause metallation. This benefical structure was effectively used by Caubere and co-workers [21] for the metallation of pyridine The situation is somewhat better in $n$-BuLi assisted metallation, at least to some degree, but the yields remain low. Also, $\mathrm{LiOEN}(\mathrm{Me})_{2}$ yields better results with $n$-BuLi than sec-BuLi, both in terms of yield and regioselectivity. Finally, despite the low yield, it is worth noting the remarkable selectivity witnessed when using Li-OEEBu. Although this phenomena is not understood as yet, it indicates the possibility to obtain all-ring substitution by simultaneously playing with structural and kinetic parameters.

The results obtained in the metallation of toluene by using different organolithium compounds and the most reactive ligand, i.e. LiOEM are also tabulated in Table 2 . The less basic $n$-BuLi, yields higher conversion with $100 \%$ benzylic positions metallation, whereas sec-BuLi gives some metallation at ring positions. It has also been pointed out by Broaddus [18] that decreasing base strength results in increasing reactivity at benzylic positions. If one accepts the principle that the $\mathrm{CH}$ bond will be broken to the largest extent in the transition state involving the weaker base [22], it may be reasonably proposed that electron delocalisation factors will also be largest with the weaker base, i.e. more charge is developed on carbon, therefore, delocalisation is a key factor, favouring reaction at benzylic positions relative to $\mathrm{sp}^{2} \mathrm{CH}$ protons. Another way to state this hypothesis is that an increase in base strength has less effect on the rate of reaction at benzylic position, owing to decreased effectiveness of resonance stabilisation. On the other hand, no metallation occurs with DPMLi and DPHLi, probably due to the higher steric hindrance that inhibits Li exchange with toluene proton.

The amount of PEA used in these reactions was then varied to establish the optimum PEA to organo-lithium molar ratio for both sec- and $n$-BuLi activated by LiOEM (Table 3 ).

At lower catalyst (LiOEM) concentration (0.3:1) a substantial amount of ring metallation product (up to $17 \%$ ) was produced. As the amount of PEA increases from 0.3 to 3 , the amount of toluene being kept constant (i.e. constant excess 5:1 of toluene over PEA), for both sec- and $n$-BuLi cases, yield increases parallel to the formation of benzylic position product that then reaches close to $100 \%$. The effect of the ratio of metallating agent to catalyst on the ratio of products for metallation of mesitylene has also been criticised by Klein et al. [23] and West Jones [24]. The increase in catalyst concentration causes acceleration in the rate of metallation. 
Table 3

Effect of LiOEM:RLi molar ratio on lithation of toluene using Rlis activated by LIOEM ${ }^{a}$

\begin{tabular}{lllll}
\hline Organolithium (RLi) & PEA:Rli (mol\%) & Yield $(\mathrm{mol} \%)$ & \multicolumn{2}{l}{ GC after reaction with $\mathrm{CH}_{3} \mathrm{I}$} \\
\cline { 4 - 5 } & & & Benzylic & Ring \\
\hline sec-BuLi & $0.3: 1.0$ & 6.0 & 35.0 & 65.0 \\
& $1.0: 1.0$ & 14.0 & 94.0 & 6.0 \\
$n$-BuLi & $3.0: 1.0$ & 46.0 & 98.5 & 1.5 \\
& $0.5: 1.0$ & 2.5 & 83.0 & 17.0 \\
& $1.0: 1.0$ & 17.0 & 89.0 & 11.0 \\
& $3.0: 1.0$ & 43.0 & 98.0 & 2.0 \\
\hline
\end{tabular}

${ }^{a}$ Reaction time, 24h; toluene:PEA, 5:1 in sec-BuLi case; toluene:Rli, 5:1 in n-BuLi case. Reaction temperature, $20{ }^{\circ} \mathrm{C}$; LiOEM.

Table 4

Effect of RLi:toluene molar ratio on lithation of toluene using $n$-BuLi activated by LiOEM ${ }^{a}$

\begin{tabular}{|c|c|c|c|}
\hline \multirow{2}{*}{$\begin{array}{l}n- \\
\text { BuLi:toluene } \\
\text { (molar ratio) }\end{array}$} & \multirow[t]{2}{*}{$\begin{array}{l}\text { Yield } \\
(\mathrm{mol} \%)\end{array}$} & \multicolumn{2}{|c|}{$\begin{array}{l}\text { GC after reaction with } \\
\mathrm{CH}_{3} \mathrm{I}\end{array}$} \\
\hline & & Benzylic & Ring \\
\hline $1.0: 1.0$ & 6.5 & 100.0 & - \\
\hline $1.0: 5.0$ & 43.0 & 98.0 & 2.0 \\
\hline $1.0: 15.0$ & 73.0 & 100.0 & - \\
\hline
\end{tabular}

${ }^{a}$ Reaction time, $24 \mathrm{~h}$; reaction temperature, $20^{\circ} \mathrm{C}$; LiOEM: $n$-BuLi, 3:1 (molar ratio)

When the catalytic reaction is slow (lesser amount of catalyst), it has been observed that ring metallation becomes competitive. For the system studied here, the 3:1 composition with highest yield and regioselectivity, was used for all subsequent experiments.

It is worth recalling that, $n$-BuLi alone can metallate toluene. However, the $0.2 \%$ yield value is not much greater than that required to established definitely that benzyl lithium is formed [25] Anyhow, the above results support the view that LiOEM plays a key role in the metallation, since the extent of the reaction and its selectivity are strongly dependent on the LiOEM concentration.

The influence of the molar ratio of organolithium to toluene on the aforementioned values was also studied, as reported in Table 4.

As the amount of substrate increases, a substantial increment in yield can be observed whereas regioselectivity stays almost constant (ca. 100\% benzylic position metallation).

Table 5

Conditions and results upon quenching with $\mathrm{CH}_{3}$ I after various time intervals ${ }^{a}$

\begin{tabular}{llll}
\hline $\begin{array}{l}\text { Reaction } \\
\text { time }(\mathrm{min})\end{array}$ & $\begin{array}{l}\text { Yield } \\
(\mathrm{mol} \%)\end{array}$ & $\begin{array}{l}\text { GC after reaction with } \\
\mathrm{CH}_{3} \mathrm{I}\end{array}$ \\
\cline { 2 - 4 } & & Benzylic & Ring \\
\hline 5 & 5.4 & 100 & - \\
13 & 43.3 & 100 & - \\
55 & 72.0 & 100 & - \\
135 & 84.4 & 100 & - \\
1440 & 75.0 & 100 & - \\
\hline
\end{tabular}

${ }^{a}$ LiOEM:n-BuLi:toluene, 3:1:15 for each case.

The influence of time was also investigated on the metallation process of toluene conducted in the presence of the $n$-BuLi/LiOEM system, for a constant reactant molar ratio $(3: 1: 15)$ for which the optimum results were achieved. Table 5 shows the conditions and results upon quenching the product with methyl iodide after various time intervals. 
It is clearly seen that reaction begins early, and that yield values increase with time. But an extension of reaction time from about 2-24 h, while not influencing the regioselectivity, promotes a small decrement in yield, maybe due to secondary reactions. Indeed, one of the primary concerns in a mechanistic study of a metallation systems must be the possibility that kinetic versus thermodynamic processes are operative. Thus, Benkeser et al. [3] has shown that metallation of aromatic substrates can be followed by the reaction of the first-formed products with an excess substrate (transmetallation) to give more stable organometallic species. However, it has been later shown that this subsequent transmetallation reaction occurs slowly relative to the initial process during metallation of toluene [26] and olefinic substrates [27,28]. The data presented in Table 5 suggests that such a kinetic versus thermodynamic competition might cause this small decrement in yield. In other words, it can be safely said that rearrangement of the first-formed organometallic species is not involved during the first few hours.

The effect of reaction temperature on the metallation process of toluene in the presence of the $n-\mathrm{BuLi} / \mathrm{LiOEM}$ system, for which the optimum results were achieved, was finally investigated. The conditions and results upon quenching the product with methyl iodide are given in Table 6.

Table 6

Effect of reaction temperature on lithiation of toluene with n-BuLi activated by LiOEM ${ }^{a}$

\begin{tabular}{llll}
\hline $\begin{array}{l}\text { Temperature } \\
\left({ }^{\circ} \mathrm{C}\right)\end{array}$ & Yield (\%) & $\begin{array}{l}\text { GC after reaction with } \\
\mathrm{CH}_{3}\end{array}$ \\
\cline { 3 - 4 } & & Benzylic & Ring \\
\hline 20 & 75.0 & 100 & - \\
0 & 36.0 & 100 & - \\
-38 & 0.90 & 100 & - \\
-78 & 0.08 & 100 & - \\
\hline
\end{tabular}

${ }^{a}$ Reaction time, 24 h; LiOEM:n-BuLi:toluene, 3:1:15

Upon increase in reaction temperature from -78 to $20{ }^{\circ} \mathrm{C}$, the benzylic metallation yield increases drastically while keeping the same regioselectivity high. It has also been previously reported that [29] increasing of the reaction temperature from room to that of the refluxing solvent has a remarkable effect on the metallating power of base (from an average charge of 1.3 at room temperature to 2.7 at reflux) in benzylic metallations whereas metallation of allylic moieties at elevated temperatures produces side reactions.

\section{Conclusions}

It appears that particular polyether lithium alkox-ides, when combined with lithium alkyls, represent a new type of superbase, displaying interesting activity and excellent versatile selectivity profile for the metallation of alkylbenzenes. That behaviour still increases the interest they had already aroused in the field of "living" nucleophilic polymerisation of alkyl (meth) acrylates [13].

\section{Acknowledgements}

The authors are deeply indebted to the "Services Fédéraux des Affaires Scientifiques, Techniques et Culturelles" for a general grant.

\section{References}

[1] A.A. Morton, L.E. Claff, F.W. Collins, J. Org. Chem. 20 (1955) 428.

[2] D.J. Bryce Smith, J. Chem. Soc. (1954) 1079.

[3] R.A. Benkeser, J. Hooz, T.V. Liston, A.E. Trevillyon, J. Am. Chem. Soc. 85 (1963) 3984

[4] R.A. Benkeser, A.E. Trevillyon, J. Hooz, J. Am. Chem. Soc. 84 (1962) 4971.

[5] A.I. Shatenstein, Adv. Phys. Org. Chem. 1 (1963) 155.

[6] A. Streitwieser, R.A. Caldwell, R.G. Lawler, G.R. Ziegler, J. Am. Chem. Soc. 87 (1965) 5399.

[7] J.E. Hofmann, A. Schrieshiem, R.E. Nichols, Tetrahedron Lett. (1965) 1745.

[8] L. Lochmann, D. Lim, J. Paspisil, Tetrahedron Lett. (1966) 2.

[9] J. Hartman, M. Schlosser, Helv. Chim. Acta 59 (1976) 453.

[10] M. Schlosser, J. Org. Chem. 18 (1967) 9.

[11] M. Schlosser, S. Strunk, Tetrahedron Lett. 25 (1964) 741.

[12] R. Lehmann, M. Schlosser, Tetrahedron Lett. 25 (1984) 745.

[13] J.S. Wang, R. Jérôme, Ph. Teyssié, Macromolecules 27 (1994) 4896.

[14] L. Lochmann, J. Petranek, Tetrahedron Lett. 32 (11) (1991) 1483. 
Published in: Journal of molecular catalysis A: chemical (2002), vol. 179, iss. 1-2, pp. 59-64

Status: Postprint (Author's version)

[15] Fr. Hein, E. Petzchner, K. Wagler, Fr.A. Segitz, Z. Anorg. Allgem. Chem. 141 (1924) 204.

[16] K. Ziegler, F. Dersch, Berchet 64 (1931) 448.

[17] H. Gilman, Org. Reactions 8 (1957) 258.

[18] D. Broaddus, J. Org. Chem. 35 (1970) 10.

[19] G.G. Eberhardt, W.A. Butte, J. Org. Chem. 29 (1964) 2928.

[20] H.D. Froehlich, P. Scholz, V. Griehl, H. Stoyl, E. Anton, VEB Chemiche Werke Buna Ger. (East) DD 256, 519 (Cl. CO7F1/02), May

1988, Appl. 299, 418, 23 January 1987.

[21] P. Gros, Y. Fort, P. Caubere, J. Chem. Soc., Perkin Trans. 120 (1997) 3597.

[22] J.E. Leffler, E. Grunwald, Rates and Equilibria of Organic Reactions, Wiley, New York, 1963, p. 158.

[23] J. Klein, A. Medlik, A. Meyer, Tetrahedron Lett. 32 (1976) 51.

[24] R. West, P.C. Jones, J. Am. Chem. Soc. 90 (1968) 2656.

[25] H. Gilman, H.A. Pacevitz, O.J. Baine, Am. Chem. Soc. 62 (1940) 1514.

[26] C.D. Broaddus, J. Am. Chem. Soc. 88 (1960) 4174.

[27] C.D. Broaddus, J. Org. Chem. 29 (1964) 2689.

[28] C.D. Broaddus, D.L. Muck, J. Am. Chem. Soc. 89 (1967) 6533.

[29] B. Gordon, J.E. Loftus, J. Org. Chem. 51 (1986) 1618. 